\title{
A new digital spectrograph for observations of radio burst emission from the Sun
}

\author{
E. Ebenezer, R. Ramesh, K. R. Subramanian, M. S. SundaraRajan, and Ch. V. Sastry \\ Indian Institute of Astrophysics, Bangalore 560 034, India
}

Received 4 August 2000 / Accepted 4 December 2000

\begin{abstract}
A new digital spectrograph for obtaining a dynamic spectrum of radio burst emission from the Sun in the frequency range $30-80 \mathrm{MHz}$ has been recently commissioned at the Gauribidanur Radio Observatory (Lat: $13^{\circ} 36^{\prime} 12^{\prime \prime} \mathrm{N}$ and Long: $77^{\circ} 27^{\prime} 07^{\prime \prime} \mathrm{E}$ ), about $100 \mathrm{~km}$ north of Bangalore, India. This paper describes various aspects of the antenna system, frontend receiver and digital hardware of the spectrograph. Some of the initial results obtained with the instrument are also presented.
\end{abstract}

Key words. Sun: Corona; radio radiation - instrumentation: spectrograph

\section{Introduction}

The Indian Institute of Astrophysics recently began operating a new digital spectrograph at the Gauribidanur radio observatory (Sastry 1995), to observe radio burst emission from the Sun in the frequency range $30-80 \mathrm{MHz}$. It works on the principle of measuring the autocorrelation function of the input signal, and then Fourier transforming it to obtain its power spectrum. To our knowledge, this is the first time a solar radio spectrograph based on the above principle is implemented. This instrument is expected to play a useful role, particularly during the maximum of the present solar cycle (Cycle 23), since we have the unique opportunity to also locate the position of the burst sources using two-dimensional images obtained with the existing Gauribidanur radioheliograph (GRH, Ramesh et al. 1998) in the frequency range $40-150 \mathrm{MHz}$.

\section{Antenna system}

The basic receiving element used in the present system is a Log periodic dipole (LPD) which has an almost continuous coverage of a wide range of frequencies. Its effective collecting area is about $0.5 \lambda^{2}$, and has a characteristic impedance of $\approx 50 \Omega$. The dipoles are made of aluminium tubes and are designed to operate in the frequency range $40-150 \mathrm{MHz}$ with a VSWR $<2$, and a directional gain of $8 \mathrm{~dB}$. The measured half-power beamwidths of the LPD are approximately $60^{\circ}$ in the E-plane and $100^{\circ}$ in the Hplane, enabling observations to be carried out over a wide range of hour angle and declination (Ramesh 1999).

Send offprint requests to: R. Ramesh,

e-mail: ramesh@iiap.ernet.in

\section{Receiver system}

\subsection{Analog frontend}

At present, the R.F. signal from one of the antenna groups in the east-west arm of the GRH forms the input to the spectrograph. The group consists of 8 LPDs with an interelement spacing of $10 \mathrm{~m}$. The elements are oriented in the east-west direction and they accept linear polarisation in that direction (Fig. 1). The R.F. output from each element is passed through a high pass filter and then amplified in a broadband amplifier of noise figure $\approx 300 \mathrm{~K}$. The high pass filter is used to cut off large interfering signals at frequencies $<40 \mathrm{MHz}$ which otherwise might give rise to spurious intermodulation products. In addition, it also reduces the dynamic range requirements on the subsequent stages of electronics. The various individual LPDs in the group are connected using RG8U cables and power combiners in a Christmas tree fashion, as shown in Fig. 2, and the combined output is again amplified in a broadband amplifier before being transmitted via open-wire lines (attenuation of about $0.5 \mathrm{~dB} / 100 \mathrm{~m}$ at $150 \mathrm{MHz}$ ) to the central receiver building. We use balanced-to-unbalanced (BALUN) transformers to connect the R.F. signal at both ends of the open-wire line. The measured VSWR of the transformers is $\approx 1.5$ at $100 \mathrm{MHz}$.

In the receiver building, the R.F. signal from the field goes through a series of amplification and mixing operations before fed into the digital receiver. It is first upconverted to an intermediate frequency (I.F.) of $170 \mathrm{MHz}$. This conversion places all the image frequencies well above the frequency of observation. The different R.F. signals are selected by sweeping the first local oscillator (L.O.) to different frequencies from 200 to $250 \mathrm{MHz}$, in steps 


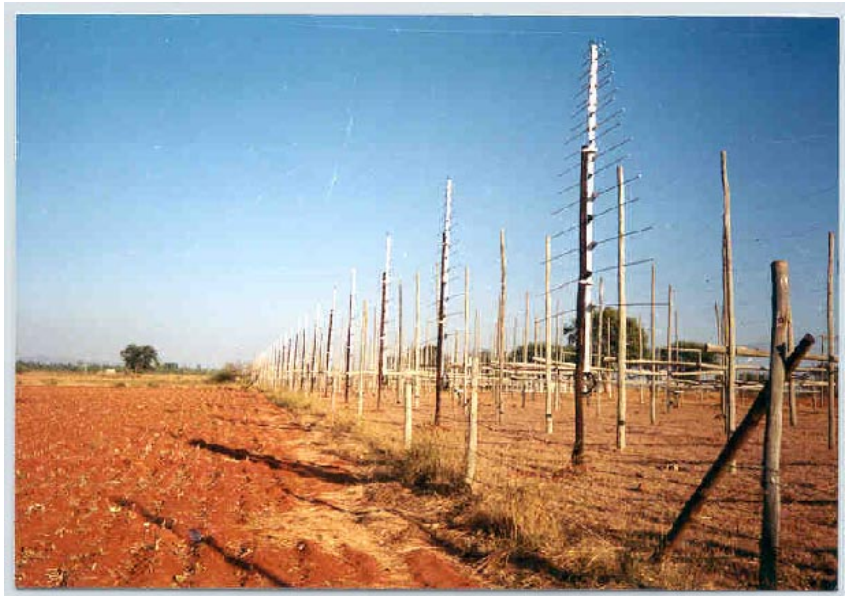

Fig. 1. East-West arm of the GRH

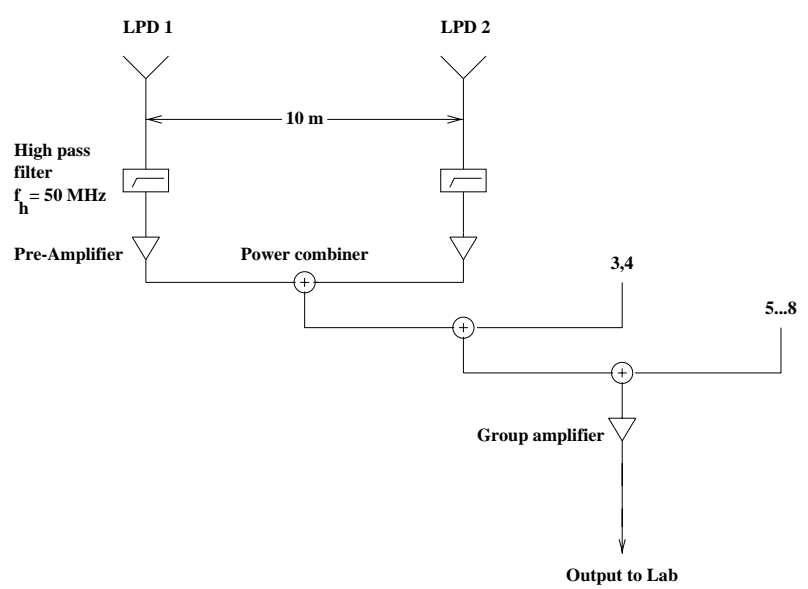

Fig. 2. Array configuration

of $1 \mathrm{MHz}$. The dwell time at each frequency is about 64 msec. The I.F. signal is amplified and passed through a bandpass filter with a center frequency $\left(f_{\mathrm{c}}\right)$ of $170 \mathrm{MHz}$ and a bandwidth $(\Delta f)$ of $6 \mathrm{MHz}$ to suppress the possible spurious pick-ups through harmonics. Then it is downconverted to a 2nd I.F. of $10 \mathrm{MHz}$ by mixing with a fixed L.O. of $180 \mathrm{MHz}$. The output is again amplified and passed through a band pass filter with $f_{\mathrm{c}}=10 \mathrm{MHz}$ and $\Delta f=1 \mathrm{MHz}$ to minimize contributions from unwanted signals at other frequencies. Figure 3 shows the schematic of the analog frontend reciever. The present minimum detectable flux density of the system is about $2000 \mathrm{Jy}$ $\left(1 \mathrm{Jy}=10^{-26} \mathrm{~W} / \mathrm{m}^{2} / \mathrm{Hz}\right.$ ) at $f=80 \mathrm{MHz}$, and for an integration time of $64 \mathrm{msec}$. This enables us to carry out observations of radio emission from the "quiet" Sun also (Fig. 7).

\subsection{Digital backend}

In the digital receiver, the $10 \mathrm{MHz}$ I.F. signals are first quantised to two levels using a zero crossing detector (AD 790). Its output is a TTL signal corresponding to whether the input I.F. signal is below the "ground" level or above it. The quantised signal is sampled (the sampling

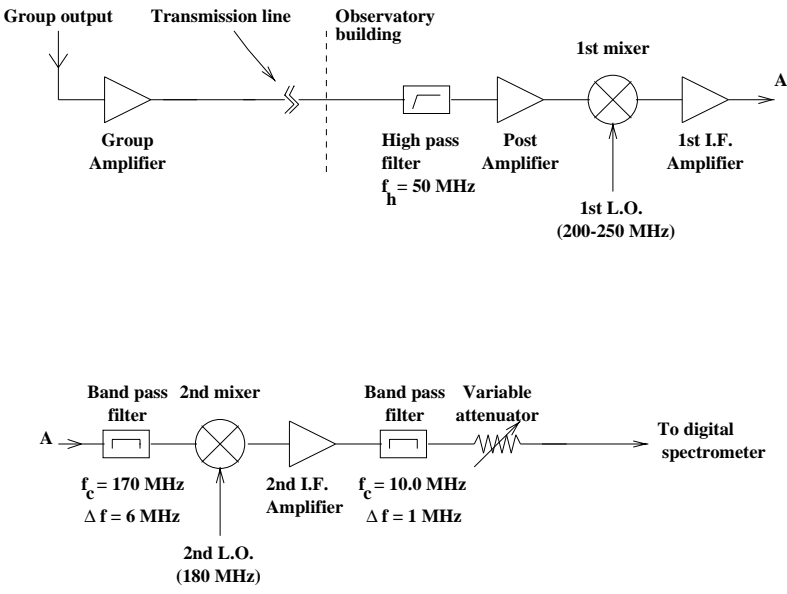

Fig. 3. Schematic diagram of the analog frontend receiver

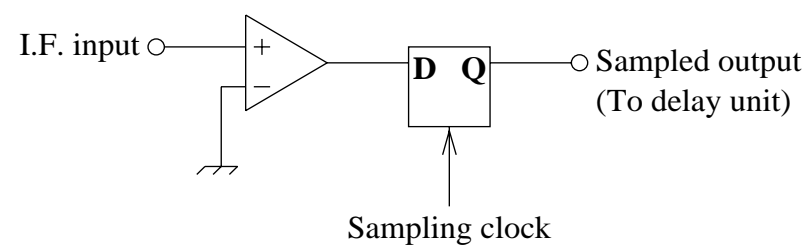

Fig. 4. Block diagram of the 1-bit digitizer

rate used in the present case is $2 \mathrm{MHz}$ ) in a dual D-type positive edge triggered flip-flop (74LS74), and its output is then passed through a delay circuit. Figure 4 shows the block diagram of the digitizer (quantiser + sampler) used in the spectrograph. We note here that the signal-to-noise ratio in a correlator with a 1-bit, 2-level quantizer is only $64 \%$, compared to the analog case (Van Vleck \& Middleton 1966). However, the loss in sensitivity is not severe particularly in the case of solar observations, since the strength of the observed signal is strong (Nakajima et al. 1993). The loss in the absolute amplitude information due to the infinite clipping of the input signal in the zero crossing detector can also be regained (Udayashankar \& Ravishankar 1990).

\subsubsection{Delay set-up}

As mentioned earlier, the power spectrum of the input signal in the present case is estimated by measuring its autocorrelation function (Weinreb 1963). To carry out this, the sampler output is fed to a set of two 8-bit serial-shift registers (74LS164) which converts each sampled data into a 16-bit stream (Boxes S-A \& B-J in Fig. 5). The shift registers are clocked at the sampling rate, and the 8 th bit of the first unit (Box A in Fig. 5) is taken as the reference. This implies that the adjacent data bits lag/lead each other by $0.5 \mu \mathrm{sec}$, and the various delay values range from $-7 \tau$ to $+8 \tau$, where $\tau=0.5 \mu$ sec. This arrangement gives a frequency resolution of $\approx 125 \mathrm{KHz}$ in the final power spectrum. 


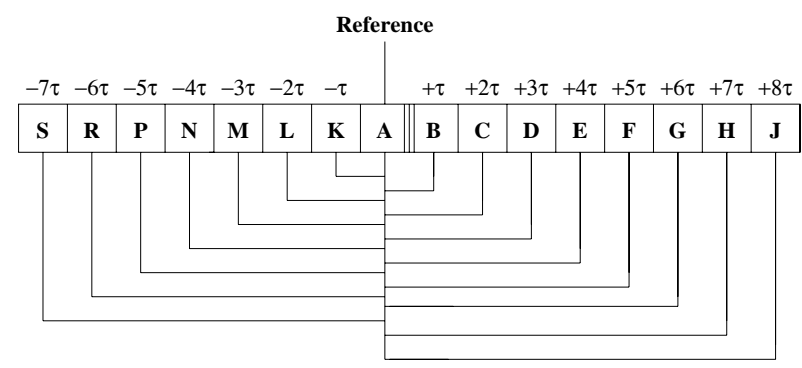

Fig. 5. Delay setup

\subsubsection{Correlator}

The correlator chip used in our spectrograph was basically designed for measuring the complex visibility of a radio source in an interferometer array. These are custom built chips using the CMOS gate array technology. The architecture of the chip is shown in Fig. 6. The output of the cosine correlator is $\mathrm{C} 1 \oplus \mathrm{C} 2+\mathrm{S} 1 \oplus \mathrm{S} 2$ and that of the sine correlator is $\mathrm{C} 1 \oplus \mathrm{S} 2-\mathrm{C} 2 \oplus \mathrm{S} 1$, where $\mathrm{C} 1, \mathrm{C} 2$ and $\mathrm{S} 1, \mathrm{~S} 2$ are the cosine and sine inputs to the correlator from the two antennas of an interferometer, respectively. In the present case, the chip is used in the autocorrelation mode, and only the first of the above two outputs from each chip is read. The sine inputs S1 \& S2 are forced to zero. As mentioned in the earlier section, the sampler output is delayed by various values ranging from $-7 \tau$ to $+8 \tau$ by passing through a set of two 8-bit shift registers. Each bit from the latter is correlated with the reference bit (the 8th bit of the first shift-register) to yield the autocorrelation function of the input signal for a particular time $t$. Likewise, the autocorrelation values corresponding to the various samples are measured, and are accumulated in a counter for a pre-determined length of time (integration time). Later, the output of the counter (the correlation count for a particular integration period) is read into the computer. The stored data is Fourier transformed offline to obtain the power spectrum of the R.F. signal selected by the L.O. Note that at any given time the spectrum is obtained for only a small bandwidth $(1 \mathrm{MHz})$. To obtain the spectrum for the entire band from $30-80 \mathrm{MHz}$, we change the L.O. to different frequencies $(200-250 \mathrm{MHz})$, as mentioned earlier.

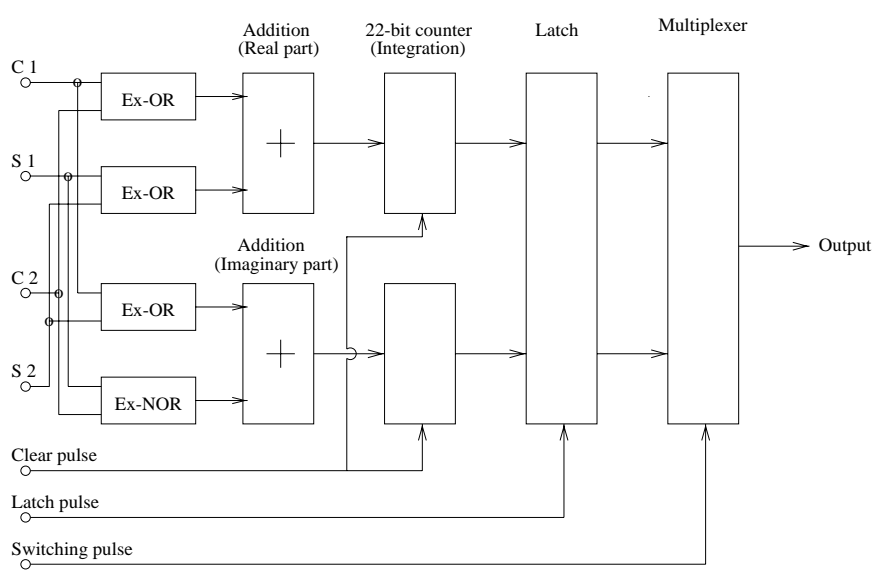

Fig. 6. An elementary circuit of the correlator chip

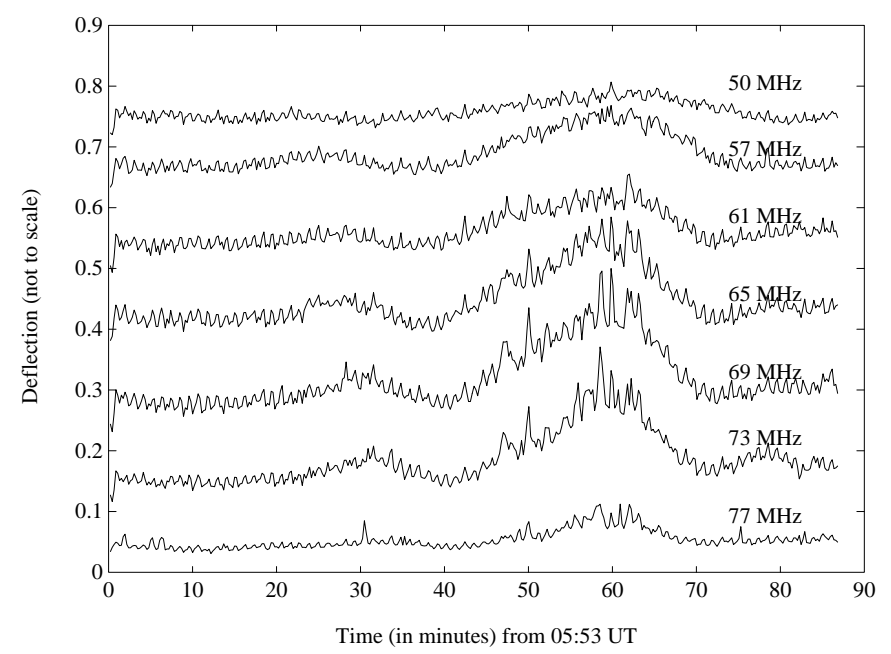

Fig. 7. Radio emission from the "quiet" Sun observed on April 4, 2000

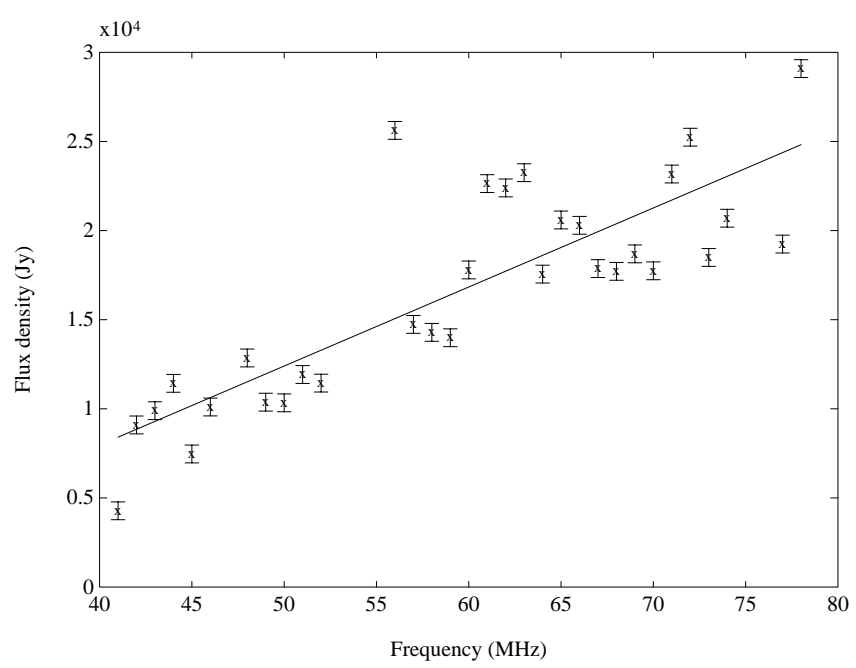

Fig. 8. Flux density of the "quiet" Sun observed on April 4, 2000 at different frequencies in the range 40-80 MHz. The error involved in the measurement is also shown. The straight line is the least squares fit to the measured values

\section{Initial results}

\section{1. "Quiet" Sun observation}

In this section, we present observations on the "quiet" Sun carried out with the spectrograph on April 4, 2000. Figure 7 shows the observed drift scan on this day at a few selected frequencies. It can be seen that the intensity rises above the background around 06:40 UT, reaches a maximum at 06:53 UT, and falls back at 07:05 UT. The Sun was "quiet", and no burst emission was seen in our data. The observations were calibrated using the cosmic radio source Cas "A", and the measured flux density at different frequencies is shown in Fig. 8. The estimated average spectral index is 2.98 . The error in the flux density values is mainly due to the difference in the declination between the Sun and the calibrator, and is expected to be 


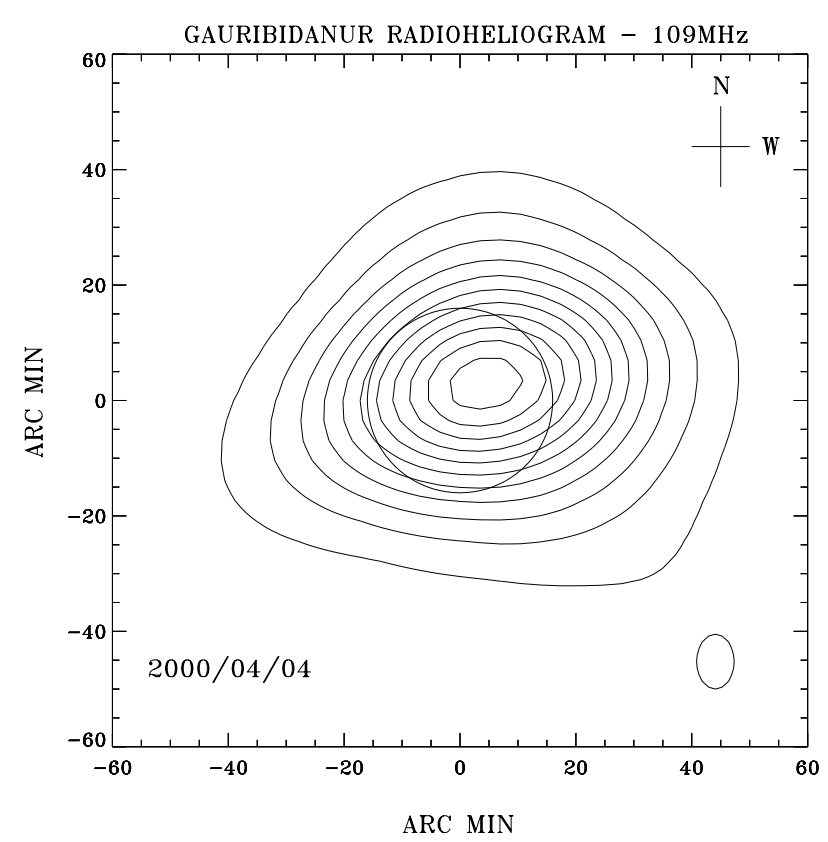

Fig. 9. Radioheliogram obtained with the GRH on April 4, 2000. The open circle at the center is the solar limb. The instrumental beam is shown at the bottom right hand corner

approximately $10 \%$ at all the frequencies. Figure 9 shows the radioheliogram obtained with the GRH on April 4, 2000 at $109 \mathrm{MHz}$. The Sun was "quiet", and no strong, localised, non-thermal sources were seen in our data. The observed peak brightness temperature was $1.210^{6} \mathrm{~K}$.

\subsection{Radio burst observation}

In this section we present a radio burst event observed on March 5, 2000 with our spectrograph. The observed dynamic spectrum on this day is shown in Fig. 10. One can clearly notice a drift in the observed intensity from 80 to $30 \mathrm{MHz}$, starting around 06:48:40 UT. The calculated drift rate $(\sim 10 \mathrm{MHz} / \mathrm{s})$ suggests that it might be a type III radio burst. Figure 11 shows the radioheliogram obtained with the GRH on March 5, 2000 around 06:50 UT.

According to the Solar Geophysical Data (April 2000), a SF/C4.2 class white-light/X-ray flare was observed on that day from 06:47-06:53 UT with a maximum at 06:51 UT. The event took place at the heliographic location S12 E41, and was associated with the active region NOAA 8898. One can notice that there is a good positional correspondence between the flare site and the intense radio source in the south-east quadrant in Fig. 11. It is possible that the latter might be the location of the radio burst in Fig. 10 since it is well established that there is a close correspondence between flares and type III radio bursts (Kundu 1965). Also no other strong, non-thermal radio source $(\mathrm{s})$ were observed on the Sun that day with the GRH. The estimated brightness temperature $\left(T_{\mathrm{b}}\right)$ of the source is about $710^{8} \mathrm{~K}$. The weak source close to the west limb in Fig. 11 is a noise storm continuum, and its $T_{\mathrm{b}}$ is $\approx 510^{6} \mathrm{~K}$.

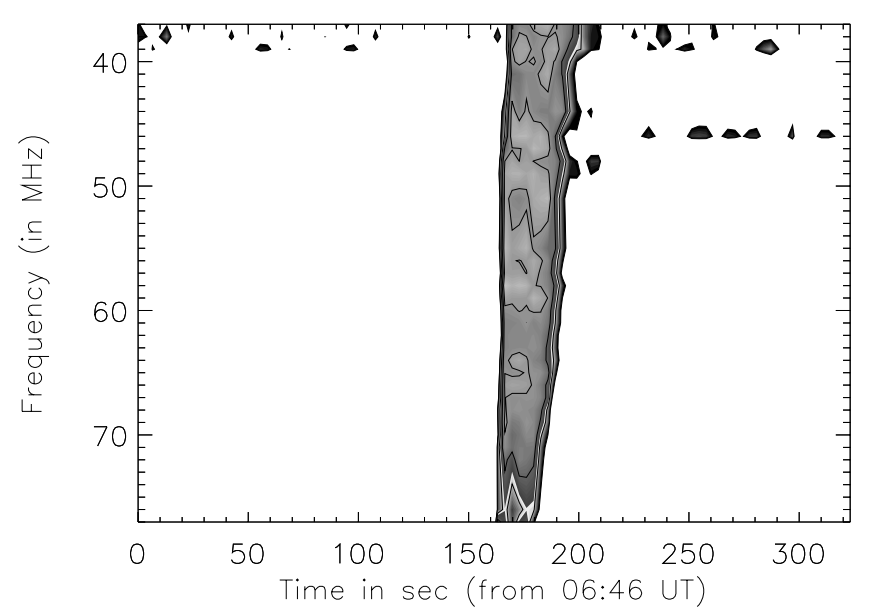

Fig. 10. Radio burst emission from the Sun observed on March 5, 2000

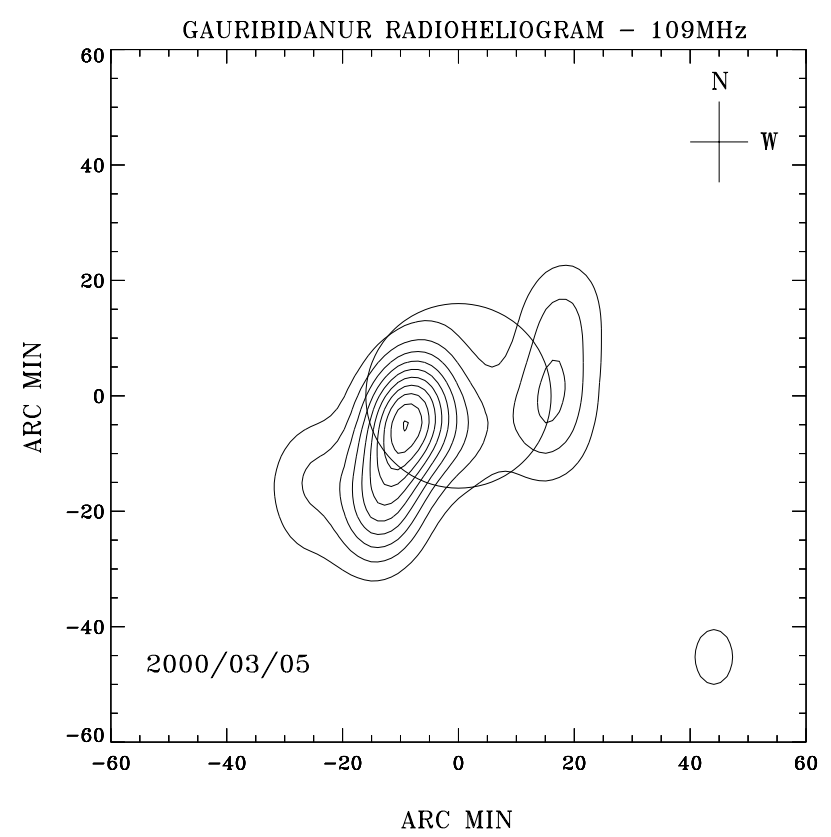

Fig. 11. Radioheliogram obtained with the GRH on March 5, 2000

\section{Present status}

As mentioned earlier, presently the spectrograph is connected to one of the antenna groups in the east-west arm of the existing heliograph in the Gauribidanur radio observatory. Work is in progress to connect the instrument to a stand alone antenna system which would allow longer observation duration ( $\approx 6 \mathrm{hrs})$. Also, it is planned to increase the bandwidth of the spectrograph to about $300 \mathrm{MHz}$. The present instrument is mainly to show that it is practically possible to obtain a dynamic spectrum of the solar radio bursts by a Fourier transformation of the autocorrelation function of the input signal. Table 1 lists the various low frequency solar radio spectrographs in operation at present (data taken from Aurass 1999). Except for the Gauribidanur instrument, all the others use an analog configuration (spectrum analysers, 
Table 1. Low frequency solar radio spectrographs in operation at present

\begin{tabular}{llll}
\hline Location & Range & \multicolumn{2}{l}{ Resolution } \\
\cline { 3 - 4 } & $(\mathrm{MHz})$ & $\begin{array}{l}\text { temporal } \\
(\mathrm{s})\end{array}$ & $\begin{array}{l}\text { spectral } \\
(\delta f / f)\end{array}$ \\
\hline Bruny Island (Australia) & $37-3$ & 3 & 0.05 \\
Culgoora (Australia) & $1800-18$ & 3 & -0.5 \\
Hiraiso (Japan) & $2500-25$ & 3 & 0.1 \\
Gauribidanur (India) & $80-30$ & 3 & 0.25 \\
IZMIRAN (Russia) & $240-45$ & 0.1 & - \\
Nancay (France) & $75-25$ & 0.1 & $>0.2$ \\
Tremsdorf (Germany) & $800-40$ & 0.1 & 0.23 \\
USAF (USA, Network) & $80-30$ & 3 & - \\
\hline
\end{tabular}

multi-channel spectrograph, sweep spectrograph, etc.) to observe radio burst emission from the Sun (Boischot et al. 1980; Mann et al. 1992; Kondo et al. 1995; Krüger \& Voigt 1995; Prestage 1995; Erickson 1997).

\section{Conclusion}

We have built a new digital radio spectrograph to obtain a dynamic spectrum of the transient burst emission from the solar corona in the frequency range $30-80 \mathrm{MHz}$ i.e., at distances of about $1.2-1.6 R_{\odot}$ from the center of the Sun. The two main advantages of the present system over the existing conventional solar radio spectrographs are: (i) it allows the user to select frequency bands which are completely free of inteference, and (ii) the desired spectral accuracy can be achieved by just changing the delay resolution in the digital receiver. This instrument is expected to play a useful role particularly during the maximum of the present solar cycle (Cycle 23) since we have the unique opportunity to also locate the position of the burst sources using two-dimensional images obtained with the GRH. A multi-wavelength collaborative study using data obtained with ground-based instruments, and those on board space missions like YOHKOH, WIND, SOHO, etc. is expected to be helpful in understanding the physics of the Sun, more clearly.

Acknowledgements. We thank Prof. R. Cowsik, Director, Indian Institute of Astrophysics for his kind support to the Gauribidanur radio astronomy project. A. T. Abdul Hameed, C. Nanje Gowda, G. N. Rajasekara, A. Anwar Saheb, and D. Babu are thanked for their help in the construction of the antenna and receiver system.

\section{References}

Aurass, H. 1999, in Proc. of the Nobeyama Symposium on Solar physics with radio observations, ed. T. Bastian, N. Gopalswamy, \& K. Shibasaki, NRO Report 479, 293

Boischot, A., et al. 1980, Icarus, 43, 399

Erickson, W. C. 1997, Publ. Astron. Soc. Aust., 14, 278.

Kondo, T., et al. 1995, J. Commun., Res., Lab., 42(1), 111

Krüger, A., \& Voigt, W. 1995, Solar Phys., 161, 393

Kundu, M. R. 1965, Solar Radio Astronomy (Interscience Publishers), 274

Mann, G., Aurass, H., Voigt, W., \& Paschke, J. 1992, in Proc. of the First SOHO Workshop, ESA SP-348, 129

Nakajima, H., et al. 1994, Proc. IEEE, 82(5), 705

Prestage, N. P. 1995, J. Atmos. Terr. Phys., 57(14), 1815

Ramesh, R., Subramanian, K. R., SundaraRajan, M. S., \& Sastry, Ch. V. 1998, Solar Phys., 181, 439

Ramesh, R. 1999, Ph.D. Thesis, Bangalore University

Sastry, Ch. V. 1995, Space Sci. Rev., 72, 629

Solar Geophysical Data, 668, Part I, April 2000

Udayashankar, N., \& Ravishankar, T. S. 1990, JA\&A, 11(3), 297

Van Vleck, J. H., \& Middleton, D. 1966, Proc. IEEE, 54(1), 2

Weinreb, S. 1963, MIT Technical Report No. 412,

Dept. Electronic Engineering 